Polymer Journal, Vol. 10, No.3, pp 307-314 (1978)

\title{
Strain Dependence of Dielectric Properties. II. Polychloroprene Vulcanizate
}

\author{
Kozo NaKaJima, ${ }^{* 1}$ Motosuke NAOKI, ${ }^{* 2}$ and Takuhei Nose ${ }^{* 1}$ \\ *1Department of Polymer Chemistry, Tokyo Institute of Technology, \\ Ookayama, Meguro-ku, Tokyo 152, Japan. \\ *2Department of Textiles and Polymers, Faculty of Technology, \\ Gunma University, Tenjin-cho, Kiryu-shi, Gunma 376, Japan.
}

(Received October 21, 1977)

\begin{abstract}
The complex dielectric constant was measured under uniaxial deformation for the $\alpha$ and $\beta$ relaxations of polychloroprene vulcanizate. The relaxation time in the $\alpha$ relaxation increases with an increasing extension ratio at a fixed temperature. This implies that the mobility of molecular motion pertaining to the $\alpha$ process decreases as an elongational strain increases. The temperature dependence of the shift factor at fixed length fitted accurately the Vogel-Fulcher-Tammann-Hesse (VFTH) equation: $-\log a_{T}=A-B /\left(T-T_{0}\right)$. However, the parameters $B$ and $T_{0}$, computed by minimizing the standard deviation between $\log a_{T}$ and the experiments for each length, depend on extension ratio. $T_{0}$ and the glass-transition temperature $T_{\mathrm{g}}$ increase as the length is increased. This gives support to the entropy theory rather than the free volume theory for the glass transition. The activation enthalpy for higher extension is always larger than that for lower extension in the temperature region where the dielectric measurements were made, but the latter exceeds the former slightly at an extremely high temperature.

The shift factor for the $\beta$ relaxation process, obtained by superposition of dielectric loss curves, decreases as the length is increased in a region far below $T_{\mathrm{g}}$. This implies that the mobility of molecular motion pertaining to the $\beta$ process increases as the elongational strain increases.
\end{abstract}

KEY WORDS Polychloroprene / Elongation / $\alpha$ Relaxation / $\beta$ Relaxation / Glass Transition / Viscosity Equation / Activation Length /

In Part I of this series, we studied the effects of uniaxial extension on the molecular motions pertaining to the $\alpha$ and $\beta$ relaxation processes for chlorinated polyethylene vulcanizate (CPE), by using the dielectric measurement. ${ }^{1}$ In a similar manner, we now present those effects for polychloroprene vulcanizate (PC) where the main chain possesses a dipole moment. A favorable feature of CPE and $\mathrm{PC}$ is the absence of the side group mode of motions; also, may be regarded as amorphous materials.

There have been many investigations for studying the effects of pressure on the glass transition and the local mode motions, but only a few papers have been presented in regard to the effects of elongational strain on the glassy state and the glass transition. ${ }^{2-6}$ The glass transition is closely related to the molecular motion pertaining to the $\alpha$ relaxation process of amorphous polymers, and the rate of the motion is inversely proportional to the relaxation time $\tau$. Therefore, research for the nature of the relaxation time, by using precise method of measurement, will enable us to understand the characteristics of the glass transition as well as those of molecular motions.

The temperature dependence of the relaxation time, i.e., the shift factor $a_{T}$, is adequately represented for many polymeric liquids by the Williams-Landel-Ferry (WLF) equation, ${ }^{7}$

$$
\begin{aligned}
-\log a_{T} & =-\log \tau(T) / \tau\left(T_{\mathrm{g}}\right) \\
& =C_{1}\left(T-T_{\mathrm{g}}\right) /\left[C_{2}+\left(T-T_{\mathrm{g}}\right)\right]
\end{aligned}
$$

where $C_{1}$ and $C_{2}$ are usually regarded as universal 
constants. $^{8}$ However, Vogel, ${ }^{9}$ Fulcher, ${ }^{10}$ and Tammann and Hesse ${ }^{11}$ have proposed an empirical (VFTH) equation,

$$
-\log a_{T}=A-B /\left(T-T_{0}\right)
$$

which is equivalent to the WLF equation with the relations: ${ }^{12}$

$$
\begin{aligned}
& C_{1}=A=B /\left(T_{\mathrm{g}}-T_{0}\right) \\
& C_{2}=T_{\mathrm{g}}-T_{0}=B / C_{1}=B / A
\end{aligned}
$$

The pressure effects on the parameters in eq 1 and 2 were studied by the dielectric measurement for CPE, and all of these depended on pressure, i.e., on volume. ${ }^{13}$ One objective of this paper is to inquire as to whether these parameters may perhaps be constant when the sample is stretched. Since the dilation accompanying the uniaxial strain is usually very small in the liquid state ${ }^{14-16}$ any variation with extension would directly reflect the variation with change in the conformational states of the chain.

\section{EXPERIMENTAL}

The polychloroprene sample, equivalent to the familiar Neoprene W, was furnished from Showa Denko Company. The vulcanization of this material was carried out with zinc oxide and magnesium oxide at $150^{\circ} \mathrm{C}$. The sample was purified by repeated swelling with benzene and toluene, and deswelling with methanol. After drying in a vacuum at $40^{\circ} \mathrm{C}$ for one week, the test specimen was cut from a sheet $0.102-\mathrm{cm}$ thick into a shape having a length of $10 \mathrm{~cm}$ and a width of $4.5 \mathrm{~cm}$. Before measurements, the specimen was relaxed at $40^{\circ} \mathrm{C}$ for two days at the maximum extension ratio of 1.91

All dielectric properties were obtained in the same apparatus as the one used earlier for studies on $\mathrm{CPE},{ }^{1}$ except for the cell whose electrodes were made of mercury-thorium alloy instead of copper. Figure 1 shows an illustration of the electrode system. The $\mathrm{Hg}-\mathrm{Th}$ alloy, whose melting temperature $T_{\mathrm{m}}^{\text {alloy }}$ was $-59^{\circ} \mathrm{C}$, was utilized to obtain favorable contact between the sample and the electrodes. At $T_{\mathrm{m}}^{\text {alloy }}{ }^{\text {, however, }}$ a contraction in volume of the alloy came about, and corrections on the effective area of the electrodes had to be made below $T_{\mathrm{m}}^{\text {alloy }}$.

After the specimen had been stretched to the

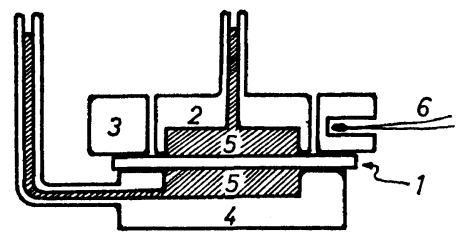

Figure 1. Illustration of the cell. The numbered parts represent: 1 , test specimen; 2 , sub-H electrode; 3 , guard electrode; 4, sub-L electrode; $5, \mathrm{Hg}-\mathrm{Th}$ electrode; 6 , thermocouple.

desired length, the stress produced was relaxed at room temperature for one day. Then the cavities of the electrodes were evaculated and the alloy was loaded. This operation was carried out each time the sample length was changed. Dielectric measurements at a fixed length were made over an extension ratio range of 1.12 to 1.91 , a frequency range of 30 to $3 \times 10^{5} \mathrm{~Hz}$, and a temperature range of -100 to $20^{\circ} \mathrm{C}$ at an interval of about $6^{\circ} \mathrm{C}$, by using a transformer bridge (Type TR10C, Ando Electric Co.).

When the dielectric measurements were finished, the test specimen was removed from the clamps and was left at room temperature until it assumed a constant length, and the distant $L_{0}$ between marked lines was measured. This recovery took about two weeks.

Since the time required to carry out all the dielectric measurements exceed two months, some deterioration on the surface of the test specimen was observed when $L_{0}$ was measured following completion of the dielectric measurements. In this paper, therefore, we do not discuss the effects of extension on relaxation intensities and the static dielectric constants, because these are easily affected by interfacial conditions between the sample and electrodes.

$\mathrm{The} \mathrm{Hg}$ - Th alloy was also employed for dilatometry instead of only $\mathrm{Hg}$, to cover the glassy region of PC and to obtain the dilatometric glass-transition temperature for the undeformed state. The specific volumes, $v_{1}$ for the liquid and $v_{\mathrm{g}}$ for the glass, are given by,

$$
\begin{gathered}
v_{1}=0.7621+4.13_{6}\left(T-287.5_{5}\right) \times 10^{-4} \quad\left(\mathrm{~cm}^{3} / \mathrm{g}\right) \\
v_{\mathrm{g}}=0.7376+1.13(T-228.5) \times 10^{-4} \quad\left(\mathrm{~cm}^{3} / \mathrm{g}\right)
\end{gathered}
$$

respectively, where temperature $T$ is in $\mathrm{K}$. The glass-transition temperature $T_{\mathrm{g}}$ is $228.5 \mathrm{~K}$ at a cooling rate of $0.3^{\circ} \mathrm{C} / \mathrm{min}$. 
Strain Dependence of Dielectric Properties. II.

\section{THE $\alpha$ RELAXATION}

\section{Superpositions}

The PC shows two peaks in dielectric loss at $300 \mathrm{~Hz}$ for all extension ratios as the temperature is varied: one at about $251 \mathrm{~K}$, above $T_{\mathrm{g}}$ of $228.5 \mathrm{~K}$, and the second at about $200 \mathrm{~K}$, in the glassy state. For the loss peaks in amorphous polymers, the relaxation above $T_{\mathrm{g}}$ are referred to as $\alpha$, and that observed immediately below $T_{\mathrm{g}}$, as $\beta$. From the isothermal plots of dielectric loss $\varepsilon^{\prime \prime}$ as a function of logarithm of frequency $\log f$, we have estimated the maximum loss $\varepsilon_{\max }^{\prime \prime}$, near $\varepsilon_{\max }^{\prime \prime}$ measurements of $\varepsilon^{\prime \prime}$ being carried out by changing $\log f$ minutely at an interval of about 0.05 .

Plots of the reduced dielectric loss, $\varepsilon^{\prime \prime} / \varepsilon_{\max }^{\prime \prime}$, for the $\alpha$ relaxation against $\log f$ for various temperatures should have given a master curve for each extension ratio. The master curves for all extension ratios fell on a master curve as shown in Figure 2, where the reference tem- perature and extension ratio are $251.7 \mathrm{~K}$ and 1.373 , respectively.

In the first paper of this series, we observed that the superpositions of $\varepsilon^{\prime \prime} / \varepsilon_{\max }^{\prime \prime}$ against $\log f$ were possible in terms of the temperature, but not in terms of the extension in CPE. This situation is not observed for the present sample of PC. All curves of $\varepsilon^{\prime \prime} / \varepsilon_{\max }^{\prime \prime}$ against $\log f$ seem to be superposable within experimental errors along the extension as well as along temperature. Although some sources of this difference in superposability between different samples, such as the influence of structual variation with extension or that of the tails of the $\beta$ relaxation, are conceivable, it is not possible from the data at our rather limited frequency range for only two samples, to determine what factor acts on the superposability in the $\alpha$ relaxation process.

The shift factor $a_{T, \lambda}$ defined by

$$
a_{T, \lambda}=\tau(T, \lambda) / \tau\left(T_{\mathrm{r}}, \lambda_{\mathrm{r}}\right)
$$

was obtained from the superpositions described

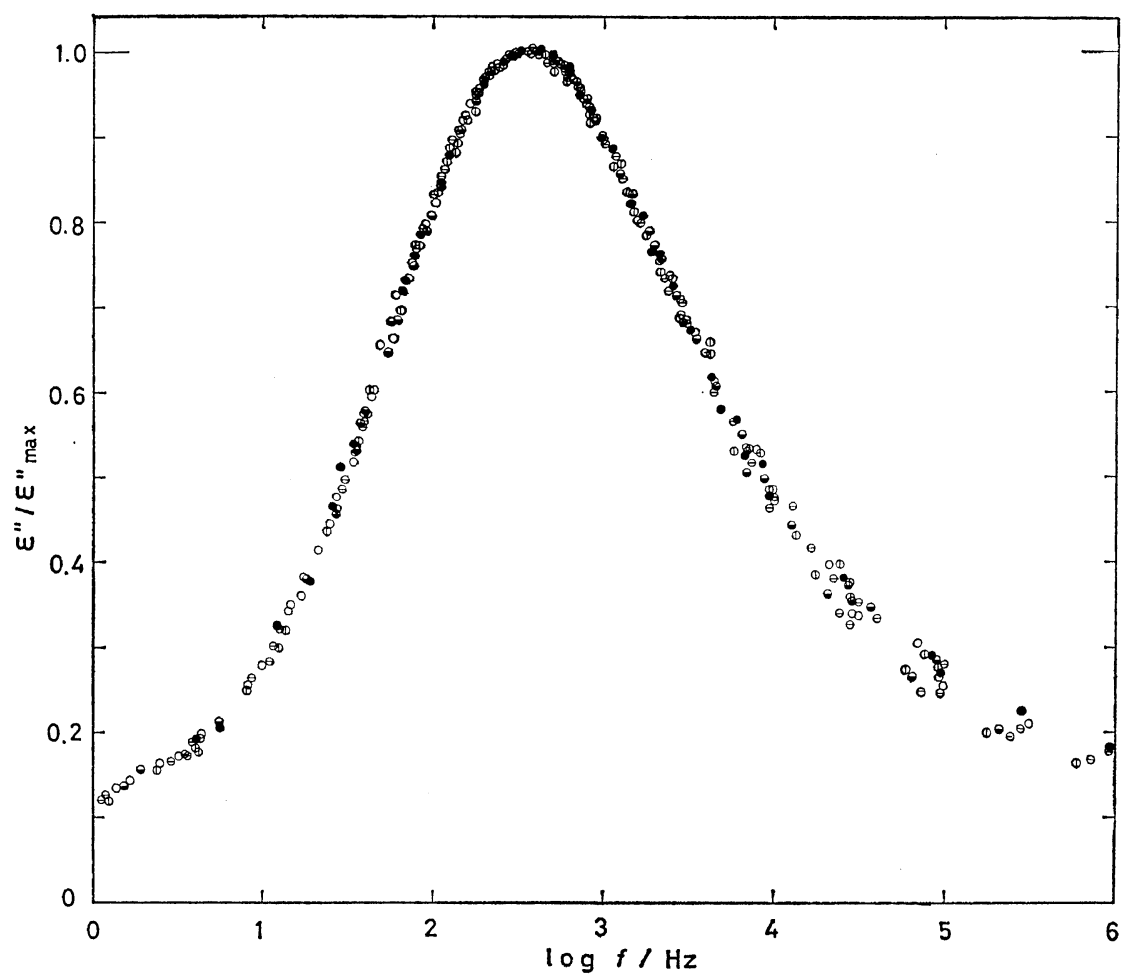

Figure 2. Master curve of $\varepsilon^{\prime \prime} / \varepsilon_{\max }^{\prime \prime}$ as a function of the logarithm of frequency for the $\alpha$ relaxation. Extension ratios at $290 \mathrm{~K}: \bigcirc, 1.122 ; \ominus, 1.373 ;(1), 1.517 ; \ominus, 1.654 ; \bigcirc, 1.911$. 


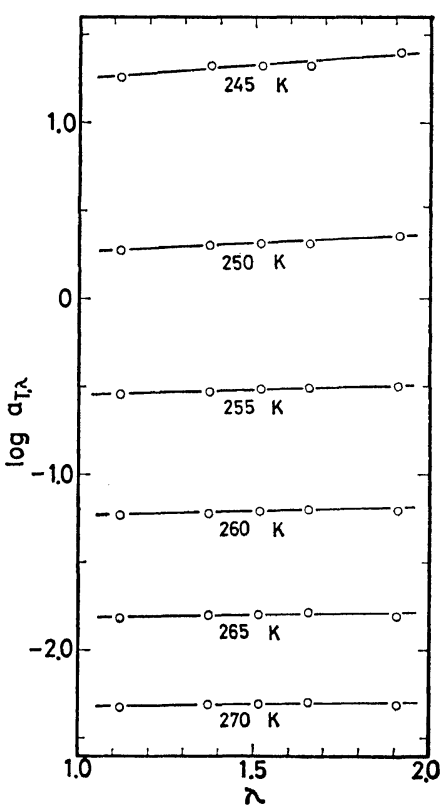

Figure 3. Isothermal variation of shift factor with extension ratio for the $\alpha$ relaxation.

above, where $T_{\mathrm{r}}$ is the reference temperature of $251.7 \mathrm{~K}$ and $\lambda_{\mathrm{r}}$ the reference extension ratio of 1.373. We use the definition $\lambda=L / L_{0}$, where $L$ is the deformed length and $L_{0}$ the undeformed one. We do not correct the thermal dilation of $L_{0}$, and $\lambda$ is the one at $290 \mathrm{~K}$ in this paper.

The isothermal shift factor as a function of extension ratio is shown in Figure 3. As the extension is increased, the shift factor increases at our temperature region. Thus the activation length defined by

$$
\begin{aligned}
\Delta L^{\neq} & =R T\left(\partial \ln a_{T, \lambda} / \partial F\right)_{P, T} \\
& =R T\left(\partial \ln a_{T, \lambda} / \partial \lambda\right)_{P, T} /(\partial F / \partial \lambda)_{P, T}
\end{aligned}
$$

which was proposed by analogy with the activation volume, ${ }^{1}$ should have a positive value. In eq $8, F$ is the tensile force. The positive sign of the activation length indicates that the stretch of the sample depresses the mobility of the molecular motion pertaining to the $\alpha$ relaxation process. The theory of rubber elasticity maintains that an increase in the elongational strain is accompanied primarily by a decrease in the conformational entropy. Therefore, the molecular motion may be inhibited by the decrease in the conformational degrees of freedom.
The slope of the shift factor against extension ratio becomes steeper as the temperature decreased. The increase in the slope suggests an increase in a conformational effect on the molecular motion with decreasing temperature.

The activation enthalpy defined by

$$
\Delta H^{\neq}=R\left[\partial \ln a_{T, \lambda} / \partial(1 / T)\right]_{P, \lambda}
$$

is shown in Figure 4 as a function of extension ratio, where $\Delta H^{\neq}(\alpha)$ refers to that in the $\alpha$ relaxation, and $\Delta H^{\ddagger}(\beta)$, the $\beta$ relaxation. $\Delta H^{\ddagger}(\alpha)$ for higher extension is always larger than that for the lower extension in our temperature region for the experiments. This situation is similar to that of $\left(\partial \log a_{T, \lambda} / \partial \lambda\right)_{P, T}$. That is, as the conformational degrees of freedom decreases, the potential energy barrier for the molecular rearrangements may increase in this temperature region.

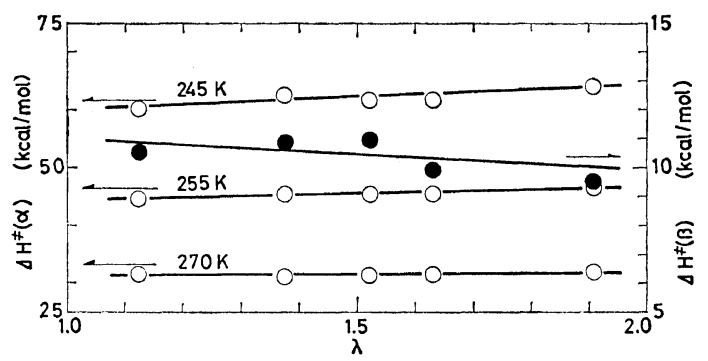

Figure 4. Isothermal variation of activation enthalpy with extension ratio for the $\alpha$ relaxation $\Delta H^{\neq}(\alpha)$, (○), and the $\beta$ relaxation $\Delta H^{\neq}(\beta),(\bigcirc)$.

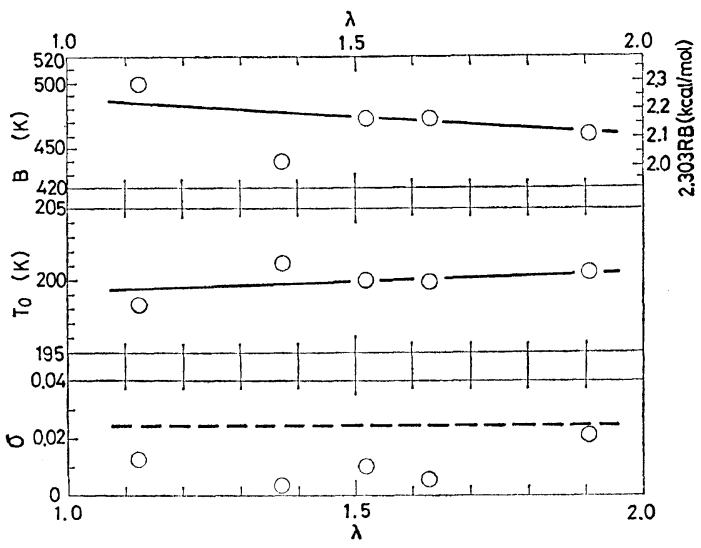

Figure 5. Variations of the VFTH parameters and standard deviation with the extension ratio. The broken line in the $\sigma v s . \lambda$ plots indicates the maximum uncertainty in superposition. 
Strain Dependence of Dielectric Properties. II.

The extension dependences of $\log a_{\mathrm{T}, \lambda}$ and $\Delta H^{f}(\alpha)$ decrease rapidly with increasing temperature and seem to vanish at a high temperature. The activation enthalpy at an extremely high temperature is available from the parameter $B$ in the VFTH equation, eq $2 . \quad B$ is regarded as the dependence of the shift factor on $T^{-1}$ at infinite temperature, and $2.303 R B$ may be regarded as the activation enthalpy at an infinite temperature. The estimation of $B$ will be shown later. It is interesting to note that the extension dependence of $2.303 R B$, shown in Figure 5, is different from that of $\Delta H^{\ddagger}(\alpha) . \Delta H^{\ddagger}(\alpha)$ increases while $2.303 R B$ decreases, as the extension ratio is increased. This may imply the presence of an inversion temperature of the extension dependence of the activation enthalpy.

\section{Glass-Transition Temperature}

In this series of papers, we define the extension dependence of the glass-transition temperature, $\mathrm{d} T_{\mathrm{g}} / \mathrm{d} \lambda$, as an extension dependence of the temperature at fixed $\tau$. Then, we have the following expressions for $\mathrm{d} T_{\mathrm{g}} / \mathrm{d} \lambda$ :

$$
\begin{aligned}
\mathrm{d} T_{\mathrm{g}} / \mathrm{d} \lambda & =(\partial T / \partial \lambda)_{P, \tau} \\
& =-\left(\partial \ln a_{T, \lambda} / \partial \lambda\right)_{P, T} /\left(\partial \ln a_{T, \lambda} / \partial T\right)_{P, \lambda}
\end{aligned}
$$

The extension dependence of the glass-transition temperature is shown in Figure 6 as a function of temperature.

The temperature variation of $(\partial T / \partial \lambda)_{P, \tau}$ is considered to correspond to the variation of the glass-transition temperature with the time scale of our observation. Therefore, when $(\partial T / \partial \lambda)_{P, \tau}$ is extrapolated to the dilatometric glass-transition temperature, as indicated by broken lines in Figure 6, we obtain the extension dependence

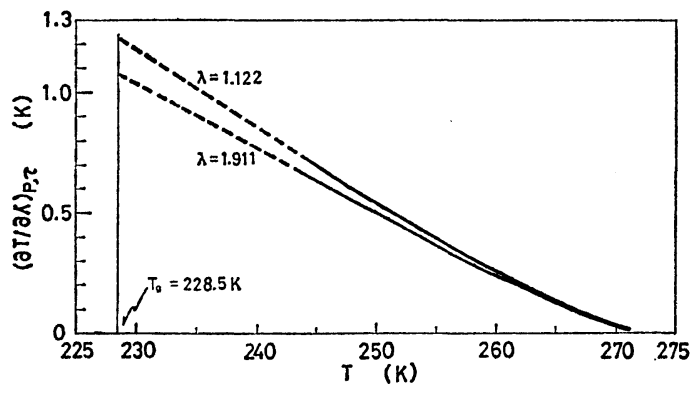

Figure 6. Extension dependence of the glass-transition temperature at a fixed length as a function of temperature. of the glass-transition temperature observed in the dilatometric time scale. This extrapolation leads to an approximate value for $\mathrm{d} T_{\mathrm{g}} / \mathrm{d} \lambda$ of $1.05 \sim 1.25 \mathrm{~K}$ at dilatometric time scale. This result agrees in sign and order of magnitude with the values, $1.6 \sim 2.0 \mathrm{~K}$ by dielectric measurement ${ }^{1}$ and $1.4 \sim 4.6 \mathrm{~K}$ by sound velocity for $\mathrm{CPE},{ }^{17}$ $1.2 \mathrm{~K}$ by dilatometry for styrene-butadiene copolymer, ${ }^{6}$ and $1.8 \mathrm{~K}$ by dilatometry for natural rubber. ${ }^{5}$ On the contrary, the results available from tensile force measurement for natural rubber and CPE show the value of $\mathrm{d} T_{\mathrm{g}} / \mathrm{d} \lambda$ to be negative. ${ }^{2,3,17}$ The cause of this difference in sign of $\mathrm{d} T_{\mathrm{g}} / \mathrm{d} \lambda$ is not clear. We only state that, from a reliable estimation by using the dielectric measurements, the sign of $\mathrm{d} T_{\mathrm{g}} / \mathrm{d} \lambda$ for $\mathrm{PC}$ and CPE is positive.

\section{Application of the VFTH Equation}

The experimental temperature dependence of $\log a_{T, \lambda}$ at fixed length was examined by using the VFTH equation, eq 2 . The parameters $A$, $B$, and $T_{0}$ were computed to minimize the standard deviation $\sigma$ between $\log a_{T, \lambda}$ and seven experimental points of $\log a_{T, \lambda}$ between 240 $300 \mathrm{~K}$ for each fixed length. The results are shown in Figure 5.

The maximum uncertainty in obtaining $\log a_{T, 2}$ by superpositions of plots of $\varepsilon^{\prime \prime} / \varepsilon_{\max }^{\prime \prime}$ against $\log f$ is about 0.024 which is indicated by the broken line in the $\sigma v s$. $\lambda$ plots of Figure 5. The standard deviations for all extension ratios lie under the line. Considering this uncertainty in superpositions and the experimental errors in the dielectric measurements, it is reasonable to state that the experimental shift factor at fixed length is adequately described by the VFTH equation, i.e., the WLF equation, in the present experimental region of temperature. The applicability of these equations to the temperatue dependence of the shift factor seems not to be affected by uniaxial deformation of the PC sample.

However, the parameters of the VFTH equation depend on the extension ratio and are not constant, though some scattering is observed, as seen in Figure 5. Thus, the parameters of the WLF equation also depend on the extension from eq 3 and 4.

$T_{0}$, sometimes considered to be identical with 
the temperature $T_{2}$ when a supercooled liquid displays a liquid-solid transformation of the second order, ${ }^{18,19}$ increases with increasing elongational strain, i.e., decreasing the conformational entropy. $T_{2}$ is defined as that temperature at which the configurational entropy vanishes. ${ }^{19}$ The configurational entropy, regarded as the difference in the entropy between the liquid and the crystal, is usually considered to consist of three terms: $:^{20}$ the conformational entropy, the communal entropy, and the entropy associated with volume change. For polymers, several authors have found that $60-80 \%$ of the entropy of fusion is the conformational entropy at the melting temperature. ${ }^{20-22}$ This effective conformational contribution to the configurational entropy should remain near $T_{2}$. Therefore, since the present result shows $T_{0}$ to increase as the conformational entropy decreases, the usually accepted idea, though without evident grounds, that $T_{0}$ corresponds to $T_{2}$, is considered quite reasonable.

When rubber is stretched, the volume slightly increases with the dilation coefficient $(\partial \ln V / \partial \lambda)_{P, T}$ of about $10^{-4} \sim 10^{-5} \cdot{ }^{14-16}$ Therefore, if $T_{0}$ is the temperature at which the free volume vanishes, ${ }^{8,23,24} T_{0}$ should decrease with increasing extension ratio. Hence the free volume approach does not give any account of the extension dependence of $T_{0}$.

The above discussion also applies to the extension dependence of the glass-transition temperature. If the free volume determines the glass transition, the glass-transition temperature may decrease with increasing extension. If the configurational entropy determines the glass transition, the glass-transition temperature may increase with increasing extension. Here again, the free volume theory cannot predict the positive dependence of the glass-transition temperature on extension in Figure 6, whereas the entropy theory can. This conclusion is the same as that given from the studies on the pressure dependence of dielectric properties for poly(vinyl acetate) ${ }^{25}$ and CPE. ${ }^{26}$

\section{THE $\beta$ RELAXATION}

Plots of the reduced dielectric loss $\varepsilon^{\prime \prime} / \varepsilon_{\max }^{\prime \prime}$ for the $\beta$ relaxation process against $\log f$ for various temperatures and lengths were superposed to give a master curve within experimental errors, as shown in Figure 7, where the reference temperature and extension ratio are $204.4 \mathrm{~K}$ and 1.122 , respectively. From these superpositions, the shift factor $a_{T, \lambda}$ for the $\beta$ process was obtained.

Arrhenius plots for the shift factor are shown in Figure 8. Although noticeable errors are observed, the shift factor decreases as the extension ratio is increased. This implies that the mobility of molecular motion pertaining to the $\beta$ relaxation process increases with increasing elongational strain.

The $\beta$ relaxation occurs for a measuring frequency of $300 \mathrm{~Hz}$ at about $0.80 T_{\mathrm{g}}$ as indicated before. This is similar to the empirical relation of Matsuoka and Ishida: ${ }^{27} T_{\mathrm{g}}{ }^{\beta} \cong 0.7 \sim 0.8 T_{\mathrm{g}}$ at $100 \mathrm{~Hz}$, which is observed approximately to hold also in rigid simple molecules. ${ }^{28}$ Assuming that $T_{\mathrm{g}}{ }^{\beta} / T_{\mathrm{g}} \cong 0.80$, we may have $T_{\mathrm{g}}{ }^{\beta}$ to be $181 \mathrm{~K}$.

The extension dependence of the shift factor increases with decreasing temperature, as seen in Figure 8. Extrapolating the extension dependence of the shift factor to $T_{\mathrm{g}}{ }^{\beta}$, we may obtain the extension dependence of $T_{\mathrm{g}}{ }^{\beta}$ with the aid of the assumption of eq 10. This leads to an approximate value for $\mathrm{d}_{\mathrm{g}}{ }^{\beta} / \mathrm{d} \lambda$ of about $-3 \mathrm{~K}$, which is negative. It is noted, however, that the differential $\mathrm{d} T_{\mathrm{g}}{ }^{\beta} / \mathrm{d} \lambda$ has no physical reality. Because the glasses for each extension ratio have been glass-formed at fixed length and the state parameters, e.g., $T$ and $\lambda$, are not continuous between the glasses for each extension ratio.

The temperature dependence of the shift factor is approximated to be the Arrhenius type within experimental error. The activation enthalpy independent on $T$ for the $\beta$ relaxation process, $\Delta H^{\mp}(\beta)$, is plotted in Figure 4 as a function of extension ratio. $\Delta H^{\ddagger}(\beta)$ slightly decreases with increasing extension ratio.

Since the density of the glass is greatly affected by the glass-transition temperature and the glassforming condition, because of the difference in the thermal expansions between the liquid and the glass, the extension effects on volume for the glass is much larger than that for the liquid. Therefore, it is not possible from our present data for only extension dependence of dielectric 


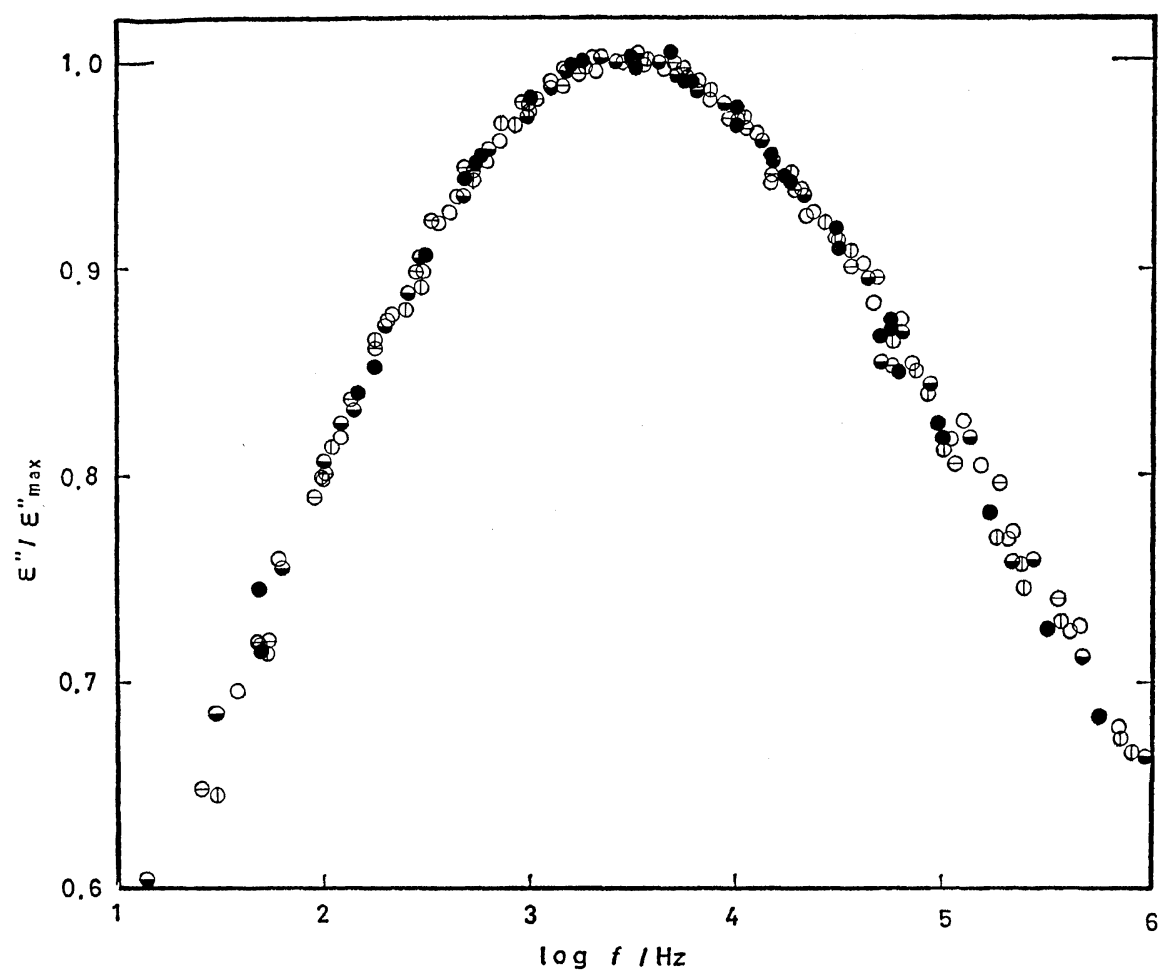

Figure 7. Master curve of $\varepsilon^{\prime \prime} / \varepsilon_{\max }^{\prime \prime}$ as a function of the logarithm of frequency for the $\beta$ relaxation. The symbols are the same as those in Figure 2.

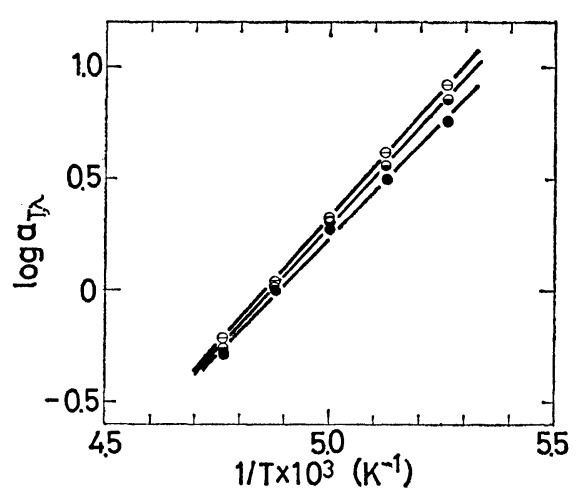

Figure 8. Arrhenius plots of extension ratio difference for the $\beta$ relaxation. The symbols are the same as those in Figure 2.

properties, to discuss the direct contribution of the conformational properties on the $\beta$ relaxation process.

Acknowledgments. Particular acknowledgments are due to Dr. T. Saito at the Research Labora- tories of Showa-Denko Company who prepared the sample studied, and to Mr. H. Nakauchi at the Research Laboratories of Bridgestone Company who carried out the vulcanization of this sample.

\section{REFERENCES}

1. M. Naoki, K. Nakajima, T. Nose, and T. Hata, Polym. J., 6, 283 (1974).

2. R. S. Witte and R. L. Anthony, J. Appl. Phys., 22, 689 (1951).

3. J. R. Stevens and D. J. Ivey, J. Appl. Phys., 29, 1390 (1958).

4. P. Mason, J. Appl. Polym. Sci., 1, 63 (1959).

5. G. Gee, P. N. Hartley, J. B. M. Herbert, and H. A. Lanceley, Polymer, 1, 365 (1960).

6. A. Komatsu and T. Nose, Rep. Prog. Polym. Phys. Jpn., 14, 309 (1971).

7. M. L. Williams, R. F. Landel, and J. D. Ferry, J. Am. Chem. Soc., 77, 3701 (1955).

8. J. D. Ferry, "Viscoelastic Properties of Polymers," 2nd ed., Wiley, New York, N.Y., 1970.

9. P. Vogel, Physik. Z., 22, 645 (1921). 
10. G. S. Fulcher, J. Am. Ceram. Soc., 8, 339 (1925).

11. G. Tammann and W. Hesse, Z. Anorg. Allgem. Chem., 156, 245 (1926).

12. See $\mathrm{p} 305$ of ref 8 .

13. M. Naoki, M. Motomura, T. Nose, and T. Hata, J. Polym. Sci., Polym. Phys. Ed., 13, 1737 (1975).

14. R. G. Christensen and C. A. J. Hoeve, J. Polym. Sci., Part A-1, 8, 1503 (1970).

15. C. Price, J.C. Padget, M. C. Kirkham, and G. Allen, Polymer, 10, 573 (1969).

16. G. Allen, M. C. Kirkham, J. C. Padget, and C. Price, Trans, Faraday Soc., 67, 1278 (1971).

17. M. Naoki and T. Nose, Polym. J., 6, 45 (1974).

18. J. H. Gibbs, J. Chem. Phys., 25, 185 (1956).

19. J.H. Gibbs and E. A. DiMarzio, J. Chem. Phys., 28, 373 (1958); ibid., 28, 807 (1958).
20. H. W. Starkweather and R. H. Boyd, J. Phys. Chem., 64, 410 (1960).

21. Y. Tsujita, T. Nose, and T. Hata, Polym. J., 3., 583 (1972).

22. A. Turturro and U. Bianchi, J. Chem. Phys., 62, 1668 (1975).

23. A. K. Doolittle, J. Appl. Phys., 22, 1471 (1951).

24. M. H. Cohen and D. Turnbull, J. Chem. Phys., 31, 1164 (1959).

25. J. M. O'Reilly, J. Polym. Sci., 57, 429 (1962).

26. M. Naoki and T. Nose, J. Polym. Sci., Polym. Phys. Ed., 13, 1747 (1975).

27. S. Matsuoka and Y. Ishida, J. Polym. Sci., Part C, 247 (1966).

28. L. Hayler and M. Goldstein, J. Chem. Phys., 66, 4736 (1977). 